\title{
Mitochondrial genome of Rhipicephalus cf. camicasi Morel, Mouchet et Rodhain, 1976 from a camel (Camelus dromedarius Linnaeus) in Riyadh, Saudi Arabia
}

\author{
Shona Chandra ${ }^{(1)}{ }^{1}$, Abdullah D. Alanazi $\mathbb{D}^{2}$ and Jan Šlapeta $\left(\mathbb{D}{ }^{1}\right.$ \\ ${ }^{1}$ Sydney School of Veterinary Science, Faculty of Science, University of Sydney, Sydney, New South Wales, Australia; \\ ${ }^{2}$ Faculty of Science and Humanities, Shaqra University, Ad-Dawadimi, Saudi Arabia
}

\begin{abstract}
Rhipicephalus camicasi Morel, Mouchet et Rodhain, 1976 is thought to be distributed across Africa, Arabian Peninsula and the Mediterranean region. It belongs to the Rhipicephalus sanguineus (Latreille, 1806) species complex. Mitochondrial genome sequences are becoming frequently used for the identification and differentiation of tick species. In the present study, the entire mitochondrial genome of $R$. cf. camicasi $(\sim 15 \mathrm{~kb})$ collected from a camel in Saudi Arabia was sequenced and compared with mitogenomes of two species of Rhipicephalus Koch, 1844. The mitochondrial genome is $87.8 \%$ and $91.7 \%$ identical to the reference genome of $R$. sanguineus (sensu stricto, former "temperate lineage") and Rhipicephalus linnaei (Audouin, 1826) (former "tropical lineage"). The current study delivers a molecular reference for material that resembles $R$. camicasi. We propose to consider the current material, including the complete mitogenome, as the reference for $R$. camicasi, until a revision using topotypical material is available.
\end{abstract}

Keywords: Rhipicephalus sanguineus complex, mtDNA, brown dog tick, phylogeny, genome, next-generation sequencing

Rhipicephalus camicasi Morel, Mouchet et Rodhain, 1976 is a tick species belonging to the Rhipicephalus sanguineus (Latreille, 1806) species complex (Morel et al. 1976, Camicas et al. 1998, Walker et al. 2000, Gray et al. 2013). Despite its wide distribution in Africa and the Arabian Peninsula, and records in the Mediterranean, $R$. camicasi remains challenging due to its close morphological resemblance with related species within the species complex and absence of genetic characterisation of either the type specimen and/or vouchers collected from the type locality (Estrada-Peña et al. 2004, Estrada-Peña 2017).

Recent studies have demonstrated its assumed molecular identity, yet there is no information about the true molecular identity of the type material (Chandra et al. 2019, Šlapeta et al. 2021). We have previously reported presence of $R$. cf. camicasi from Saudi Arabia in a morphological and genetic study (Chandra et al. 2019). The phylogenetic position was based on short sequences of $12 \mathrm{~S}$ rRNA and cox 1 genes (Chandra et al. 2019). While short PCR amplified sequences provided the initial evidence for the phylogeny of the brown dog ticks, it is becoming increasingly apparent that whole mitochondrial DNA sequences provide a far superior and robust result (Šlapeta et al. 2021). Whole genome mitochondrial DNA (mtDNA) sequences have enabled the improvement in the understanding of tick diversity for several genera as well as whole country biodiversity studies (Mans et al. 2019, Wang et al. 2019, Jia et al. 2020). Accessibility to whole genome sequencing approaches assists acquiring whole mitogenomes as well as characterisation of pathogens caried by the ticks (Ravi et al. 2019, Jia et al. 2020). It is therefore important to characterise complete mitogenomes from well-characterised reference material deposited in museums to improve taxon sampling in public nucleotide databases.

The aim of this study was to obtain the whole mtDNA sequence for $R$. cf. camicasi from Saudi Arabia from Chandra et al. (2019) and confirm its distinct phylogenetic position to related species for which the complete mtDNA sequences are known. We have achieved this by utilising whole genome sequencing and phylogenetic analyses to confirm its relationship to available known sequences of brown dog ticks.

\section{MATERIAL AND METHODS}

The Rhipicephalus cf. camicasi SC0126 sensu Chandra et al. (2019) tick was collected on a camel (Camelus dromedarius Linnaeus) in Riyadh, Saudi Arabia. Riyadh Province is an area of $404,240 \mathrm{~km}^{2}$ located in the geographic center of Saudi Arabia between $24.3800 \mathrm{~N}$ and $46.4300 \mathrm{E}$. The DNA was previously isolated from morphologically characterised voucher specimen 


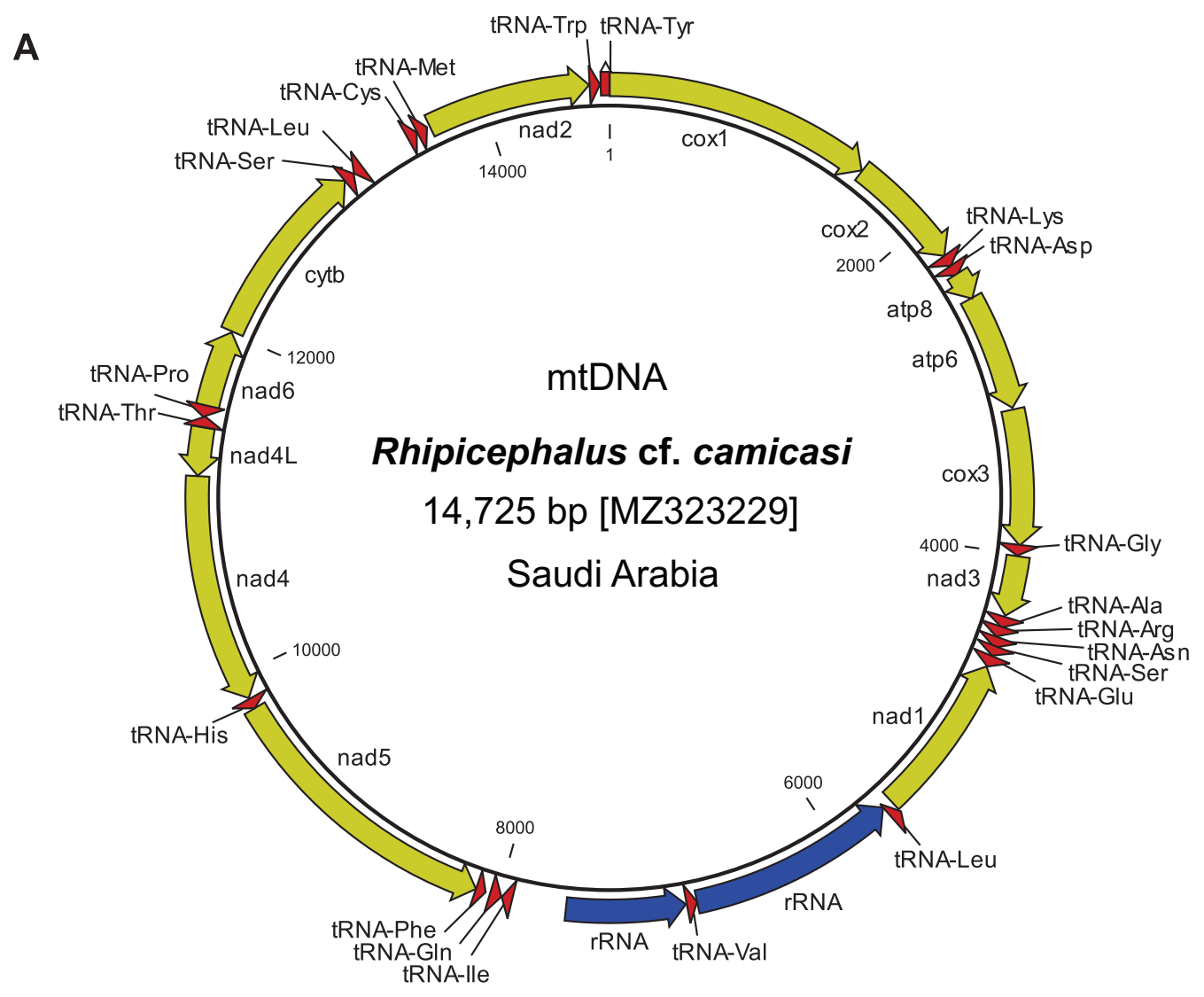

B

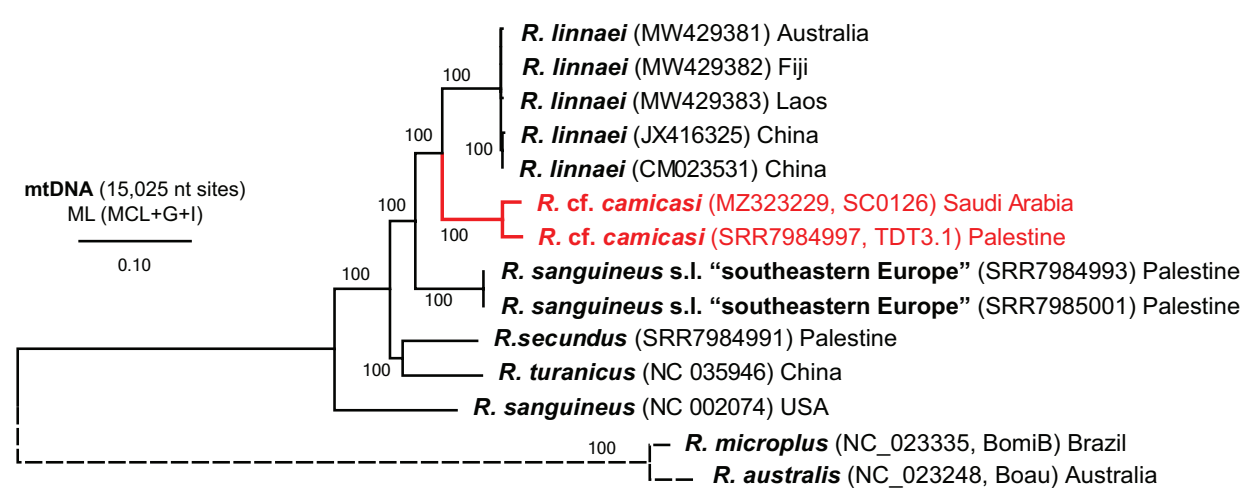

Fig. 1. Organisation of the mitochondrial genome of the tick Rhipicephalus cf. camicasi Morel, Mouchet et Rodhain, 1976. A - the mitochondrial genome (mtDNA) from the Saudi Arabian specimen (SC0126, Chandra et al., 2019) is shown including 13 protein coding genes (yellow), two rRNA genes (blue) and 22 tRNAs (red); B - phylogeny of mitochondrial genomes in species of Rhipicephalus Koch, 1844. The mtDNA phylogenetic tree based on nucleotide data (nt) was inferred by using the Maximum Likelihood method and Maximum Composite Likelihood approach with a discrete Gamma distribution among sites $(+\mathrm{G})$ and the rate variation model allowed for some sites to be evolutionarily invariable $(+\mathrm{I})$. The tree is drawn to scale, with branch lengths measured in the number of substitutions per site. Bootstrap values (200 replicates) represent the percentage of trees in which the associated taxa clustered together is shown below the branches. Evolutionary analyses including selection of models were conducted in MEGA X.

SC0126 (Chandra et al. 2019). DNA isolated from an adult $R$. cf. camicasi was used for Illumina Nextera XT library preparation followed by next-generation sequencing (NGS) using $100 \mathrm{bp}$ paired end Illumina HiSeq 2500 sequencing system at a depth of $1 \mathrm{~Gb}$ of raw sequence data (JS4291; Macrogen, Seoul, Republic of Korea) and for NEBNext ${ }^{\circledR}$ DNA Library Prep Kit followed by NGS using 150 bp paired end Illumina HiSeq 2500 sequencing system at a depth of $1 \mathrm{~Gb}$ of raw sequence data (JS6026; Novogene, Singapore).
The 150 bp paired end library sequencing was attempted, because the previous $100 \mathrm{bp}$ pair end sequencing did not yield a complete mitogenome. The whole mtDNA was assembled from FastQ data using the MITObim pipeline (Hahn et al. 2013) [https://github.com/chrishah/MITObim] with the aid of the DNA sequence obtained using cox 1 from Chandra et al. (2019).

The circular mtDNA was confirmed by the presence of overlapping ends of the assembly. The assembly was repeated three times with varying percentages of the raw FastQ sequence data (10-25\%), keeping mtDNA coverage at $60-100 \times$. The 
obtained mtDNA was annotated with the aid of MITOS Web Server (Bernt et al. 2013) [http://mitos.bioinf.uni-leipzig.de/] and aligned with available complete mtDNA sequences of species of Rhipicephalus in CLC Genomics Workbench 21 for manual validation, which included the mitogenome of a presumed $R$. camicasi (SRR7984997, TDT3.1 from Tubas, Palestine). The mtDNA phylogenetic tree based on nucleotide (nt) alignment $(15,025$ aligned sites of the entire mitogenome) was inferred by using the Maximum Likelihood method and Maximum Composite Likelihood approach with a discrete Gamma distribution among sites $(+G)$ and the rate variation model allowed for some sites to be evolutionarily invariable $(+\mathrm{I})$. Evolutionary analyses including selection of models were conducted in MEGA X (Kumar et al. 2018) [https://www.megasoftware.net/].

Raw FastQ sequence data were deposited at SRA NCBI BioProject: PRJNA733525. The nucleotide sequence data generated in this study were deposited in GenBank (NCBI);MZ323229. All sequence data, associated supplementary material and additional data are available at LabArchives (http:// dx.doi.org/10.25833/ergx-6772).

\section{RESULTS}

The Rhipicephalus cf. camicasi SC0126 sensu Chandra et al. (2019) whole genome sequencing of the $100 \mathrm{bp}$ pair end library yielded 11,407,258 total reads equaling 1.2 GB of raw data of which Q30 were 92.3\% with GC content of $45.6 \%$. The whole genome sequencing of the $150 \mathrm{bp}$ pair end library yielded $6,887,818$ total reads equaling 1.0 GB of raw data of which Q30 were 93.3\% with GC content of $45.8 \%$. Initially, the assembly of the $R$. cf. camicasi mitogenome using 100 bp pair reads yielded a maximum of $\sim 9 \mathrm{~kb}$ long contig representing an incomplete mtDNA. The assembly of the $150 \mathrm{bp}$ pair reads, however, yielded 14,725 bp long contig that was a complete, circular mtDNA. The overlapping mtDNA regions using both libraries were $100 \%$ identical. The circular mtDNA of $R$. cf. camicasi encodes 13 protein coding genes, two rRNA genes and 22 tRNAs (Fig. 1A). The previously obtained partial sequences for 12S rRNA (MH094506) and cox 1 (MH094481) by Chandra et al. (2019) from the same tick specimen (SC0126) were 100\% identical with the complete mtDNA.

The new mitogenome sequence was appended to the mtDNA alignment of all currently available Rhipicephalus sanguineus sensu lato mtDNA sequences (Šlapeta et al. 2021). The nucleotide pairwise comparison revealed that mtDNA $R$. cf. camicasi SC0126 is $96.7 \%$ identical with mtDNA $R$. camicasi (SRR7984997, TDT3.1 from Tubas, Palestine). The newly obtained mitochondrial genome sequence of the reference genome of $R$. sanguineus (sensu stricto, former "temperate lineage") and Rhipicephalus linnaei (Audouin, 1826) (former "tropical lineage") is $87.8 \%$ and $91.7 \%$ identical. Phylogenetic inference confirmed monophyly of $R$. cf. camicasi (SC0126) with $R$. cf. camicasi (TDT3.1). The two mtDNA sequences for assumed " $R$. camicasi" clade that is a sister to the clade of R. linnaei mtDNA sequences (Fig. 1B).

\section{DISCUSSION}

The type specimens of Rhipicephalus camicasi were recovered from Randa, Djibouti on a sheep and the species is considered to be distributed in the steppe and desert climatic regions of north-eastern Africa, including the Sudan, Ethiopia, Somalia and Kenya, and some countries in the Middle East such as Yemen, Saudi Arabia, Jordan and Lebanon (Morel et al. 1976, Estrada-Peña 2017). The species $R$. camicasi represents an example of tick species with ambiguous morphology within the Rhipicephalus sanguineus complex. Morphological identity is increasingly coupled with molecular identity, as a consequence of integrated approaches to defining the biodiversity. Ideally, the characterisation of the type material using molecular tools can be achieved, but often the material is lost or not available for destructive procedures for DNA isolation. To confirm the true identity of such species, examination and/ or collection of extant material that can be unambiguously linked to the type material - ideally material from the type host and type location - is preferred.

In previous studies from Saudi Arabia, R. camicasi was observed on two native sheep in a western province and camels from Riyadh Province (El-Azazy and Scrimgeour 1997, Alanazi et al. 2019). In addition, Harrison et al. (2015) reported $R$. camicasi from Acomys dimidiatus (Cretzschmar) collected from the National Wildlife Research Centre (NWRC) in Taif (western Saudi Arabia). Conservatively, we previously proposed to call the Saudi Arabian ticks $R$. cf. camicasi that we characterised morphologically, and PCR amplified partial 12S rRNA and cox1 genes (Chandra et al. 2019). We demonstrated that the Saudi Arabian $R$. cf. camicasi formed a monophyletic clade with two unpublished sequences, one $R$. camicasi from a camel in Ethiopia (FJ536556) and one Rhipicephalus sp. from an unknown host in Portugal (FJ536574). Furthermore, we have assembled the complete mitochondrial sequence from a tick next generation sequencing data (SRR7984997) from Palestine collected from a dog and suggested that it is part of the suspected $R$. camicasi clade (Šlapeta et al. 2021). Morphological identity of these ticks remains elusive except for the Saudi Arabian specimens whose morphology has been studied using SEM (Chandra et al. 2019).

Despite the limited molecular evidence, we now have two complete mtDNA sequences for this genetically defined clade that is distinct from all others within the $R$. sanguineus species complex. These complete mitochondrial sequences will serve to elucidate the diversity of the complex and contribute to the growing number of mitochondrial tick species being currently generated, and replacing the past approach based on partial 12S rRNA, 16S rRNA and cox 1 gene sequences.

On one hand, the species $R$. camicasi requires revision of the original material and new material collected from the type locality in Djibouti, to confirm its morphological and molecular identity. On the other hand, the current study delivers the molecular reference for material that morphologically resembles $R$. camicasi and was collected from camel, i.e., the known host of $R$. camicasi (see Mo- 
rel et al. 1976, Chandra et al. 2019). Until such revision is available, we propose to consider that the current material including the complete mtDNA is $R$. camicasi.

Acknowledgements. This research did not receive any specific grant from funding agencies in the public, commercial, or notfor-profit sectors.
Author contribution. Shona Chandra: designed research, performed research and wrote the paper; Abdullah D. Alanazi: performed research and contributed research material; Jan Šlapeta: designed research, contributed analytical tools, analysed data and wrote the paper. All authors read and approved the final manuscript.

\section{REFERENCES}

Alanazi A.D., Al-Mohammed H.I., Alyousif M.S., Said A.E., Salim B., Abdel-Shafy S., Shatpan R.M. 2019: Species diversity and seasonal distribution of hard ticks (Acari: Ixodidae) infesting mammalian hosts in various districts of Riyadh Province, Saudi Arabia. J. Med. Entomol. 56: 1027-1032.

el-Azazy O.M., Scrimgeour E.M. 1997: Crimean-Congo haemorrhagic fever virus infection in the western province of Saudi Arabia. Trans. R. Soc. Trop. Med. Hyg. 91: 275-278.

Bernt M., Donath A., Juhling F., Externirink F., Florentz C., Fritzsch G., Putz J., Middendorf M., Stadler P.F. 2013: MITOS: improved de novo metazoan mitochondrial genome annotation. Mol. Phylogenet. Evol. 69: 313-319.

Camicas J.-L., Hervy J.-P., Adam F., Morel P.C. (Eds.) 1998 Les Tiques du Monde (Acarida, Ixodida). Nomenclature, Stades Décrits, Hôtes, Répartition. Orstom, Paris, 233 pp.

Chandra S., Smith K., Alanazi A.D., Alyousif M.S., Emery D., Slapeta J. 2019: Rhipicephalus sanguineus sensu lato from dogs and dromedary camels in Riyadh, Saudi Arabia: low prevalence of vector-borne pathogens in dogs detected using multiplexed tandem PCR panel. Folia Parasitol. 66: 007.

Estrada-Peña A. 2017: Rhipicephalus camicasi Morel, Mouchet and Rodhain, 1976. In: A. Estrada-Peña, A.D. Mihalca, T. Petney (Eds.), Ticks of Europe and North Africa: A Guide to Species Identification. Springer International Publishing, Cham, Switzerland, pp. 317-319.

Estrada-Peña A., Bouattour A., Camicas J.-L., Walker A.R. (EDs.) 2004: Ticks of Domestic Animals in the Mediterranean Region: A Guide to Identification of Species. University of Zaragoza, Zaragoza, Spain, 131 pp.

Gray J., Dantas-Torres F., Estrada-Pena A., Levin M. 2013: Systematics and ecology of the brown dog tick, Rhipicephalus sanguineus. Ticks Tick Borne Dis. 4: 171-180.

Hahn C., Bachmann L., Chevreux B. 2013: Reconstructing mitochondrial genomes directly from genomic next-generation sequencing reads - a baiting and iterative mapping approach. Nucl. Acids Res. 41: e129.

Harrison A., Robb G.N., Alagaili A.N., Hastriter M.W., Apanaskevich D.A., Ueckermann E.A., Bennett N.C. 2015: Ectoparasite fauna of rodents collected from two wildlife research centres in Saudi Arabia with discussion on the implications for disease transmission. Acta Trop. 147: 1-5.

Jia N., Wang J., Shi W., Du L., Sun Y., Zhan W., Jiang J.F., Wang Q., Zhang B., Ji P., Bell-Sakyi L., Cui X.M., Yuan
T.T., Jiang B.G., Yang W.F., Lam T.T., Chang Q.C., Ding S.J., Wang X.J., Zhu J.G., Ruan X.D., Zhao L., Wei J.T., Ye R.Z., Que T.C., Du C.H., Zhou Y.H., Cheng J.X., DAI P.F., Guo W.B., Han X.H., Huang E.J., Li L.F., Wei W., GaO Y.C., Liu J.Z., Shao H.Z., Wang X., Wang C.C., Yang T.C., Huo Q.B., Li W., Chen H.Y., Chen S.E., Zhou L.G., Ni X.B., Tian J.H., Sheng Y., Liu T., Pan Y.S., Xia L.Y., Li J., Tick G., Microbiome C., Zhao F., CaO W.C. 2020: Largescale comparative analyses of tick genomes elucidate their genetic diversity and vector capacities. Cell 182: 1328-1340 e1313.

Kumar S., Stecher G., Li M., Knyaz C., Tamura K. 2018. MEGA X: Molecular evolutionary genetics analysis across computing platforms. Mol. Biol. Evol. 35: 1547-1549.

Mans B.J., Featherston J., Kvas M., Pillay K.A., de Klerk D.G., Pienaar R., de Castro M.H., Schwan T.G., Lopez J.E., Teel P., Perez de Leon A.A., Sonenshine D.E., EgeKwu N.I., Bakkes D.K., Heyne H., Kanduma E.G., Nyangiwe N., Bouattour A., Latif A.A. 2019: Argasid and ixodid systematics: implications for soft tick evolution and systematics, with a new argasid species list. Ticks Tick Borne Dis. 10: 219-240.

Morel P.C., Mouchet J., Rodhain F. 1976: Description of $R h$ ipicephalus camicasi n. sp. (Aearidae, Ixodida) of subdesert steppes of the plain of Afar. Rev. Elev. Med. Vet. Pays Trop. 29: 337-340.

Ravi A., Ereqat S., Al-Jawabreh A., Abdeen Z., Abu Shamma O., Hall H., Pallen M.J., Nasereddin A. 2019: Metagenomic profiling of ticks: Identification of novel rickettsial genomes and detection of tick-borne canine parvovirus. PloS Negl. Trop. Dis. 13: e0006805.

Šlapeta J., Chandra S., Halliday B. 2021: The "tropical lineage" of the brown dog tick Rhipicephalus sanguineus sensu lato identified as Rhipicephalus linnaei (Audouin, 1826). Int. J. Parasitol. 51: 431-436.

Walker J., Keirans J., Horak I. (Eds.) 2000: The Genus Rhipicephalus (Acari, Ixodidae): A Guide to the Brown Ticks of the World. Cambridge University Press, Cambridge, United Kingdom, $221 \mathrm{pp}$.

Wang T., Zhang S., Pei T., Yu Z., Liu J. 2019: Tick mitochondrial genomes: structural characteristics and phylogenetic implications. Parasit. Vectors 12: 451. 